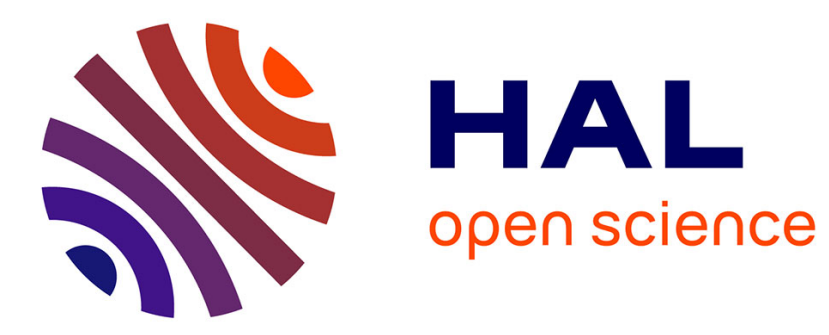

\title{
Cluster-Directed Acyclic Graph Formation for IEEE 802.15.4 in Multihop Topologies
}

Bogdan Pavkovic, Won-Joo Hwang, Fabrice Theoleyre

\section{To cite this version:}

Bogdan Pavkovic, Won-Joo Hwang, Fabrice Theoleyre. Cluster-Directed Acyclic Graph Formation for IEEE 802.15.4 in Multihop Topologies. 2012 5th International Conference on New Technologies, Mobility and Security (NTMS), May 2012, Istanbul, Turkey. pp.1-6, 10.1109/NTMS.2012.6208753 . hal-02650398

\section{HAL Id: hal-02650398 \\ https://hal.science/hal-02650398}

Submitted on 29 May 2020

HAL is a multi-disciplinary open access archive for the deposit and dissemination of scientific research documents, whether they are published or not. The documents may come from teaching and research institutions in France or abroad, or from public or private research centers.
L'archive ouverte pluridisciplinaire HAL, est destinée au dépôt et à la diffusion de documents scientifiques de niveau recherche, publiés ou non, émanant des établissements d'enseignement et de recherche français ou étrangers, des laboratoires publics ou privés. 


\title{
Cluster-Directed Acyclic Graph Formation for IEEE 802.15.4 in Multihop Topologies
}

\author{
Bogdan Pavkovic*, Won-Joo Hwang ${ }^{\dagger}$, Fabrice Theoleyre ${ }^{\S}$, \\ ${ }^{*}$ LIG, Grenoble Institute of Technology, France \\ ${ }^{\dagger}$ Computer Networks Laboratory, Inje University, Korea \\ $\S$ CNRS, LSIIT, University of Strasbourg, France \\ Email: pavkovic@imag.fr, ichwang@inje.ac.kr, theoleyre@unistra.fr
}

\begin{abstract}
IEEE 802.15.4 represents the future standard for multihop Wireless Sensor Networks. However, this protocol is based on a cluster-tree which performs poorly in multihop. In the same way, scheduling the superframes to avoid collisions represents a key challenge. We propose here a simple greedy scheduling algorithm, integrated with the IEEE 802.15.4 MAC mechanisms. This scheduling permits to modify the ClusterTree into a Directed Acyclic Graph which reduces the delay while improving robustness, removing the single point of failure problem of the original structure. Simulations proved that these mechanisms optimize the MAC layer for multihop topologies.
\end{abstract}

\section{INTRODUCTION \& MOTIVATIONS}

The IEEE has proposed a standard to govern the medium access in Wireless Sensor Networks [1]. The standard supports two main operating modes. In non-beacon mode, all the nodes use an unslotted CSMA-CA solution to access the medium. In beacon-enabled mode, the standard introduces the concept of superframes: a coordinator delimits an active part during which the other nodes use a slotted CSMA-CA to transmit their frames. Once active part is over, all the nodes may go to sleep until the next beacon periodically transmitted by the coordinator.

IEEE 802.15.4 was initially designed mainly for single hop networks: the PAN coordinator is directly connected to the end-devices, forming a star topology. Then, in a mesh topology, any pair of IEEE 802.15.4 nodes may communicate directly. Finally, in a cluster-tree, nodes forward packets along a tree rooted at the PAN coordinator. Possibly, some nodes (Reduced Function Devices) may refuse to forward packets, forming a leaf in the cluster-tree.

We will here focus on the beacon-enabled mode with a cluster-tree since this is the only way to deploy a multihop wireless sensor network while saving energy in IEEE 802.15.4. All the nodes wake-up synchronously at the beginning of the superframe: collisions between beacons are frequent. Besides, nodes buffer their packet during the sleeping period. Thus, they try to send their packets synchronously, increasing the number of collisions. Finally, the cluster-tree is a single-pointof-failure topology: a single link disconnection may degrade globally the performances.

Cuomo et al. [2] investigated the properties of the clustertree when a node associates to the first available parent. Although this study gives an overview of what we may obtain, the approach does not present a method to find the best structure. Abbagnale et al. [3] studied the impact of mobility on the topology formation process, creating some convergence problems.

Pavkovic et al. [4] proposed an opportunistic RPL version which implements QoS mechanisms. Since this scheme relies on a Directed-Acyclic Graph, the authors implemented a centralized coloring scheme to create this structure. We propose to focus here on constructing this DAG in a distributed manner to reduce the multihop problems:

1) we propose to maintain a Directed Acyclic Graph (DAG) at the MAC layer. This DAG structure optimizes both the robustness and the delay;

2) we use the Beacon-Only Period while scheduling properly the superframes to reduce collisions while limiting bandwidth wastage;

3) we present a simple localized scheduling solutions, assigning collision-free slots in a self-stabilizing manner.

\section{RELATED WORK}

\section{A. IEEE 802.15.4}

In a star, the PAN coordinator acts as gateway and collects all the packets of sensors. The mesh topology acts in a similar way, leading on a routing protocol to forward packets. Only the cluster-tree permits multihop communications: the nodes relay the packets along a tree rooted at the gateway. Zigbee may be used for instance to assign addresses hierarchically routing the packets automatically along the tree.

In non-beacon mode, all the nodes use CSMA-CA to transmit their frames: since no synchronization is required, no node can sleep. In beacon-enabled mode, IEEE 802.15.4 uses the concept of superframes. The PAN coordinator begins to send periodically beacons to delimit the beginning of its superframe. Then, all the nodes participating to its superframe can access the medium with a slotted CSMA-CA during the Contention Access Period (CAP). When the superframe is finished, all the nodes may sleep until the next beacon.

In a cluster-tree, all the non-leaf nodes must maintain a superframe to exchange packets with their children. The active part lasts for Superframe Duration (SD) and a coordinator transmits a beacon every Beacon Interval (BI). SD and BI are respectively obtained from Superframe Order (SO) and Beacon Order (BO) parameters values. The duty cycle is given by $(B O-S O)^{-1}$.

An unassociated node must discover a coordinator either passively by waiting a beacon or actively by transmitting a 
request. The node transmits an association-request during the CAP, acknowledged by the coordinator. The node has then to retrieve its association-reply after macResponseWaitTime. A coordinator starts to transmit beacons as soon as it is associated.

\section{B. Limits of IEEE 802.15.4 in multihop WSN}

For a node, the superframe of its parent is designated as incoming and the superframe maintained by the node itself as outgoing. The standard suggests the superframe of one child and its parent are interspaced by StartTime. If the StartTime value is constant, coordinators with the same depth start synchronously their superframes. Practically, beacons collide, making the protocol inefficient.

In the literature exist two main approaches to reduce the number of collisions. In the Beacon Only Period (BOP) solution, nodes implement a TDMA approach to send their beacons: at the beginning of each superframe a few slots are dedicated to beacons [5]. While collisions are avoided during the BOP, data frames may still collide in the second part since the data part keeps on overlapping. Simulations proved performances quickly degrade since hidden terminals are frequent [6].

A second solution consists in using a variable StartTime: two nodes that have the same parent should for instance not use the same StartTime so that their superframes do not overlap [7]. If we assume that all the nodes use the same BO and SO values, finding the adequate StartTime for all of them is equivalent to scheduling the superframes with a TDMA approach. Villaverde et al. [8] have proved experimentally this approach leads to the best performances.

Koubaa et al. [5] proposed a centralized algorithm to schedule the superframes with variable superframe duration (this corresponds to a classical knapsack problem). Muthukumaran et al. propose rather a greedy distributed algorithm to pick the first free slot [9]. Rhee et al [10] presented a distributed slot assignment for a TDMA MAC in wireless sensor networks. The authors use a localized greedy algorithm to pick a free slot for the transmission. We will inspire from this approach to assign slots distributively.

Pavkovic et al have proposed an opportunistic RPL version which implements QoS mechanisms [4]. Since this scheme relies on a Directed-Acyclic Graph, the authors implemented a centralized coloring scheme to create this structure. The authors proposed also a distributed version, not evaluated in the paper. This version does not work well with large densities: beacons begin to collide with each other and no re-rescheduling was proposed to cope with bad decisions.

Although most propositions focus on the MAC mechanisms, few attention has been given to the cluster-tree construction itself. Indeed, the standard does not specify how the parent should be selected, although it greatly impacts the performances. We will explore this problem in the rest of this paper.

\section{CLUSTER-DAG FOR IEEE 802.15.4}

It is well known that a simple tree is inaccurate for wireless multihop networks: the death of one single node may render non-operational the part or whole network for a long time. We propose to use rather a Directed Acyclic Graph structure: each node may have several parents, we just forbid the apparition of loops. In a DAG, several redundant paths may exist toward the PAN coordinator. Thus, we improve the robustness while not introducing any instability problem during the convergence.

We aim at minimizing the energy consumption. Thus, we should use shortest paths routing to reduce the number of transmissions. We chose to preserve a route length stretch factor of 1: the routes length through the DAG has to be the same as in the original graph.

We will now expose how we can modify IEEE 802.15.4 to lead on a cluster-Directed Acyclic Graph structure.

\section{A. Cluster-tree Construction}

We implement the passive discovery method of IEEE 802.15.4: a node waits for beacons to become aware of already associated neighboring coordinators .

A node has to choose as coordinator one of the neighbors which are the closest to the PAN coordinator. Thus, we insert in the IEEE 802.15.4 beacons a field denoting the depth, coded in 6 bits since a diameter of 63 hops seems a realistic upper bound for an IEEE 802.15.4 network.

When a node receives a beacon and is not yet associated, it chooses the source as parent. We privilege in this way the convergence time. Besides, a coordinator which has a smaller depth in the cluster-tree often transmits first its beacons.

We then apply the classical association procedure of IEEE 802.15.4: the node sends an association-request during the CAP, acknowledged by the coordinator. The node have to wait before transmitting a data-request during the next $\mathrm{CAP}$, and the coordinator replies by an ack followed by a association-reply specifying its short address (16 bits).

\section{B. Cluster-DAG}

We now aim at authorizing multiple parents. After the node transmits its association-request, it does not go to sleep, it keep on listening other beacons to find alternative candidates.

A node $N$ receiving a beacon applies the following rules:

1) the beacon is received from an already associated parent, but its depth is strictly larger than the minimum depth of the rest of its associated parents: $N$ initiate a disassociation since we aim at using shortest hop routes;

2) the beacon comes from a non-associated parent and its depth is strictly smaller than the minimum depth of all my associated parents: $N$ engages an association by sending immediately an association-request. When the corresponding ack is received, the source is inserted in the parents list, tagged as on-going association;

3) the beacon comes from a non-associated parent and its depth is strictly equal to the minimum depth of all my associated and non-associated parents: $N$ engages an association. In this way, we avoid associating to a new 
parent if an association is already on-going with a better parent: we should reduce the number of disassociations.

In conclusion, a node always maintain a list of parents which are strictly closer to the PAN coordinator than itself. In other words, we forbid any loop by maintaining shortest hops paths.

A sub-optimal parent is removed and a disassociation procedure is engaged only when the association with a better parent is concluded. Indeed, we can intermittently receive beacons through a bad link: the association will be successful only if the quality is sufficient (a too large Packet Error Rate means in IEEE 802.15.4 the association never terminates in time).

We may also integrate here a quality metric before deciding to associate with a parent: the node will estimate the packet error rate of the radio links either passively (by measuring collisions and packet delivery ratio of beacons) or actively (by sending probes). A node would select the source as parent only if the quality is sufficient.

\section{Illustration}

We represent in figure 1 the cluster-DAG we obtained. We simulated IEEE 802.15.4 with 10 nodes, distributed on a random square. One node is randomly selected to act as PAN coordinator.

We can remark that the DAG structure permits to introduce more redundancy, except for the neighbors of the PAN coordinator: by definition, no other node is closer to the PAN coordinator.

\section{SUPERFRAME ORGANIZATION}

We adopt the approach depicted in figure 2:

1) a Beacon-Only-Period is reserved at the beginning of each superframe. When several coordinators interfere but only one has children, they may use different BOP slots in the superframe;

2) we schedule the superframes such that two interfering coordinators with children do not maintain their superframe simultaneously, i.e. they use different superframe slots.

With this solution, a node without children can share its superframe with another coordinator, it just has to maintain a different BOP slot. In this way, we waste only one BOP slot and not a whole superframe.

Our solution is distributed and self-stabilizing. The algorithm converges to a legal state whatever the initial state is. By legal state, we mean a structure without loop and in which each coordinator has at least one path to the PAN coordinator;

Finally, the solution reacts automatically to interference (self-healing property): when two interfering coordinators have children and share the same superframe / BOP slots, data packets may collide. At least one of them chooses another slot to avoid collisions among data frames.

We will now explain how we can assign BOP and superframe slots to obtain these properties.

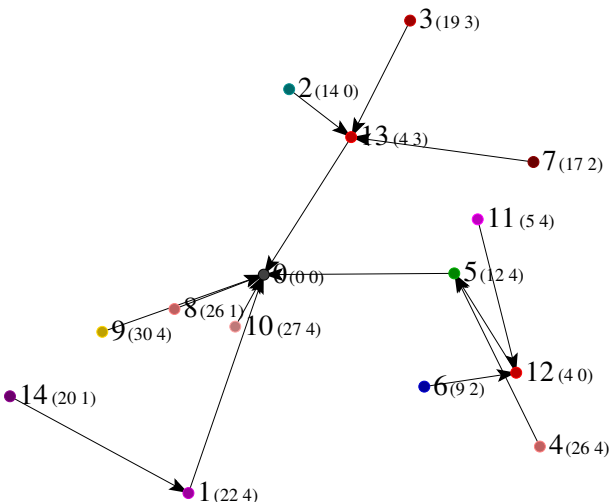

(a) Cluster tree

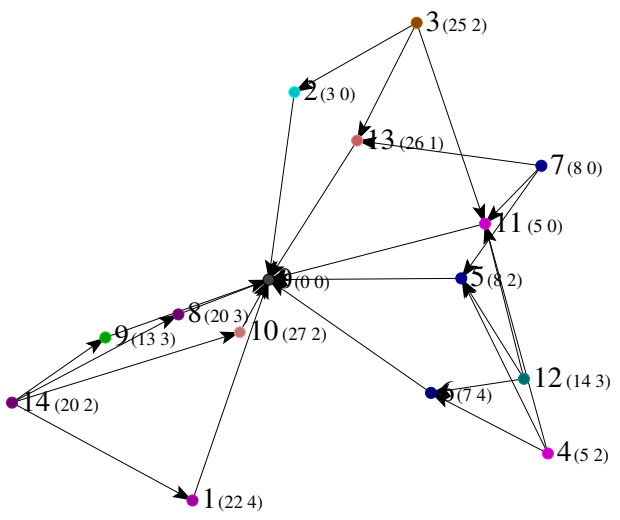

(b) DAG

Figure 1. Illustration of the topology constructed by IEEE 802.15.4- original and DAG improved version

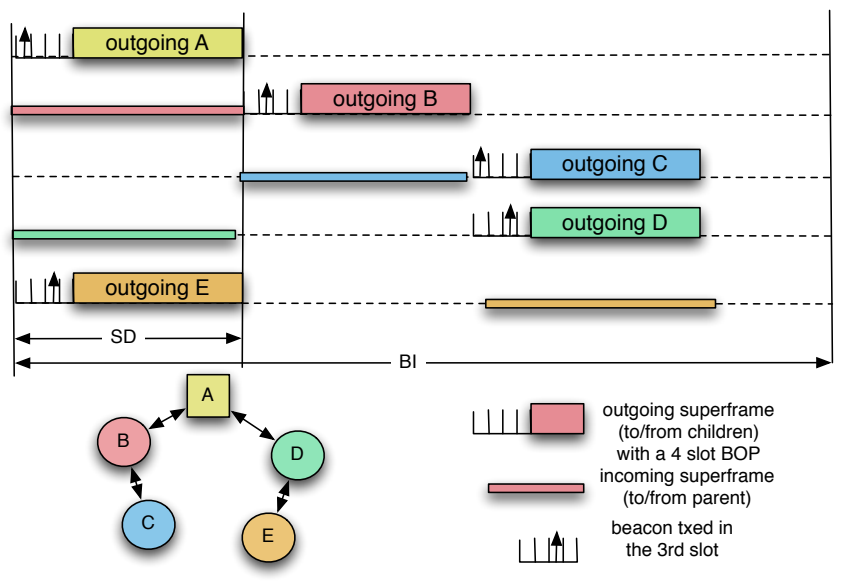

Figure 2. Beacon-Only-Period and superframe scheduling combination

\section{A. Information collect}

We aim at assigning the BOP and superframe slots while avoiding collisions. Thus, we should maintain the list of interfering nodes and their slot choice.

In IEEE 802.15.4, a node is deaf to the superframes it does not participate to. Thus, we propose that node MUST follow all the superframes maintained by neighboring coordinator, whatever its role is (parent, child, simple neighbor). As soon as a node has received the beacon, it may go to sleep if it 
does not aim at participating to this superframe.

We include in the IEEE 802.15 .4 beacons:

- the depth: distance in hops to the PAN coordinator;

- the number of children;

- a list of 1-neighbors with for each node its short address, the BOP and superframe slots they use.

To track topology changes, a node MUST periodically scan the medium to receive beacons from unknown neighbors. We may adopt the trickle timer [11] approach: the period increases when a node does not detect a new neighbor during the scanning phase (i.e. the network has converged). In this way, we limit the energy consumption due to this scanning phase.

\section{B. BOP slot Assignment}

A node selects its BOP slot in the same way as [5]: a new coordinator selects randomly one BOP slot, and listens to the medium to detect a coordinator already owner of this BOP slot. If it is free, it transmits its beacon, else it chooses another BOP slot.

\section{Superframe slot Assignment}

We propose two different algorithms to assign superframe slots: random and greedy.

In the random approach, a node chooses randomly one slot among the least loaded slots and discard the slots used by its parents to avoid collisions.

For the greedy solution, the node $N$ applies the following rules:

1) if several slots with no interfering node exist, $N$ chooses randomly one of them;

2) if no slot is empty, $N$ sorts them according to the number of interfering nodes with children. Then, it chooses randomly among the least loaded slots.

Even if this algorithm tries to limit collisions, two nodes might choose the same superframe slot at the same time. If they have not chosen the same BOP slot, they would be able to receive their respective beacons. If such collision is detected, each coordinator selects another superframe slot with a probability of $50 \%$.

If both nodes have the same superframe and BOP slots, the beacons will collide. Thus, no child will be able to associate to these coordinators. We chose that each coordinator without child re-selects another superframe slot (this coordinator did not receive any association-request and has no association-reply in its buffer). This reassignment has no consequence since no child is following this coordinator. When a coordinator starts its superframe, it may decide to change its superframe slot. In that case, it sends a beacon with its new slot: a receiver knows consequently the coordinator has changed its superframe slot. The receiver will update its neighborhood table and will follow the coordinator during its new slot: a node listens to the beacons of a coordinator even if it is not a child since it must maintain a neighborhood table.
Table I

DEFAULT PARAMETERS VALUES

\begin{tabular}{|c|c|}
\hline Radio range & 30 \\
Interference range & 60 \\
avg. nb of neighbors & 8 \\
nb nodes & 50 \\
\hline
\end{tabular}

\begin{tabular}{|c|c|}
\hline Inter packet time & $100 \mathrm{~s}$ \\
SO & 2 \\
BO & 7 \\
BOP slots & 4 \\
\hline
\end{tabular}

\section{Illustration}

Let examine the scheduling obtained in figure 1. We assume $\mathrm{BO}=7$ and $\mathrm{SO}=2$ (i.e. we have $2^{5}=32$ superframe slots) and 5 BOP slots. To each vertex, the label $X(Y, Z)$ denotes for $\mathrm{X}$ the id, $\mathrm{Y}$ the superframe slot and $\mathrm{Z}$ its BOP slot. For instance, the sink (address 0) has the superframe slot 0 and BOP slot 0 . We can verify that although slots have been assigned distributively, no collision occurs if we assume only 2-neighbors can interfere.

\section{Performance Evaluation}

We have implemented the beacon-enabled mode of IEEE 802.15.4 in the WSNet simulator [12]. We use a fixed radiorange and nodes are placed randomly in a disk to limit side effects. Default parameters are represented in tab. I. We simulated a duty-cycle comprised between $1 \%\left(2^{9-2}\right)$ and $25 \%$ $\left(2^{4-2}\right)$. We evaluated low-density topologies since we consider a power control solution should limit interference. A larger density would mean a larger topology redundancy, and thus more possibilities to choose the parents. Besides, our solutions uses the Beacon-Only Period to reduce interferences among coordinators. Thus, a small density stresses our solution.

We measured mainly the Packet Delivery Ratio (ratio between the number of transmitted packets and the number of received packets), the end-to-end delay and the superframe collision ratio (the ratio of coordinators which have an interfering coordinator sending a superframe at the same instant). We use the energy consumption values of the popular CC1100 chipset (31mA in TX, 15mA in RX and 400nA when sleeping).

We implemented 3 solutions for comparison:

1) 802.15.4: the slot used by a coordinator follows directly thus of its parent;

2) random: a coordinator chooses a random slot, except the slot(s) used by its parent(s);

3) greedy: a coordinator selects a slots not used in its neighborhood and tries to detect and solve collisions, as highlighted in section IV-C.

\section{A. Traffic model \& routing}

We model a bidirectional traffic: a node generates one packet for the PAN coordinator every $T_{\text {interpk }}$. Inversely, the PAN coordinator generates packets toward a random destination in the network with a rate $\frac{T_{\text {interpk }}}{n b_{\text {nodes }}-1}$ to obtain the same rate in the upload and download directions.

Each node maintains two buffers. The first one is a FIFO, dedicated to packets toward the PAN coordinator. A packet is pulled from the buffer when the node is in the idle state, during the CAP of the superframe of its parent. The second buffer is dedicated to the download direction: packets are extracted 


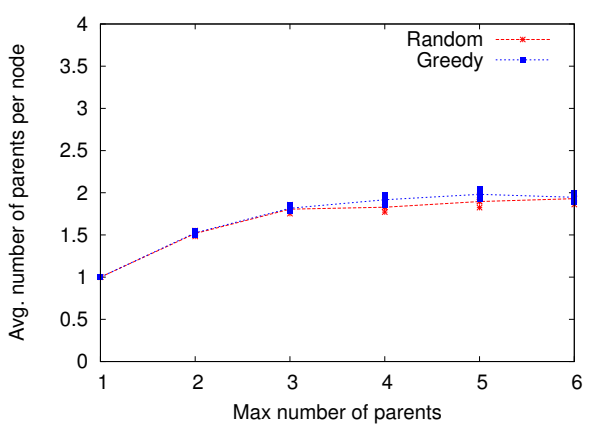

(a) DAG redundancy

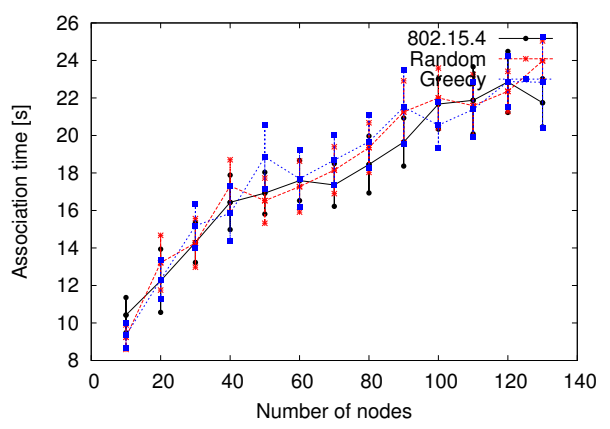

(b) Convergence time

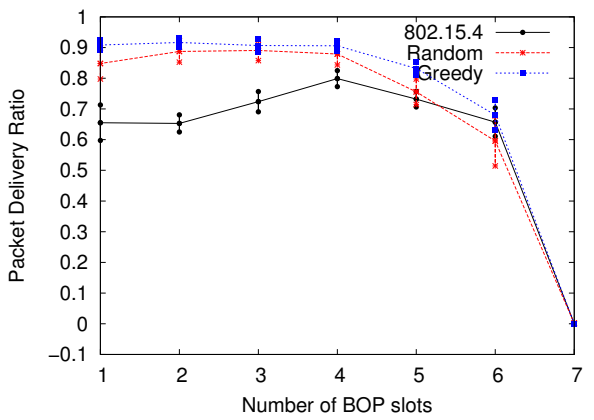

(c) BOP slots

Figure 3. Structure of IEEE 802.15.4 - $\mathrm{BO}=7, \mathrm{SO}=2$

from the buffer after the reception of a data-request from the destination. The packets for the same destination follow also the FIFO strategy (the oldest packet is transmitted first). A procedure periodically removes packets that exceeded their timeout (macTransactionPersistenceTime as defined in IEEE 802.15.4). A node which receives a beacon notifying pending packets in the coordinator MUST send a data-request before transmitting the data packets present in its upload buffer.

We implemented a centralized routing solution: packets are routed along the IEEE 802.15.4 structure. If IEEE 802.15.4 leads on a DAG, we chose to implement an anycast strategy: the node picks a packet in its upload buffer whatever the current parent is, balancing the load among all its parents.

\section{B. Cluster-DAG properties}

We first evaluated in figure 3 the structure itself. The Directed Acyclic Graph authorizes to maintain multiple parents for each node. We can remark in fig.3(a) that the redundancy
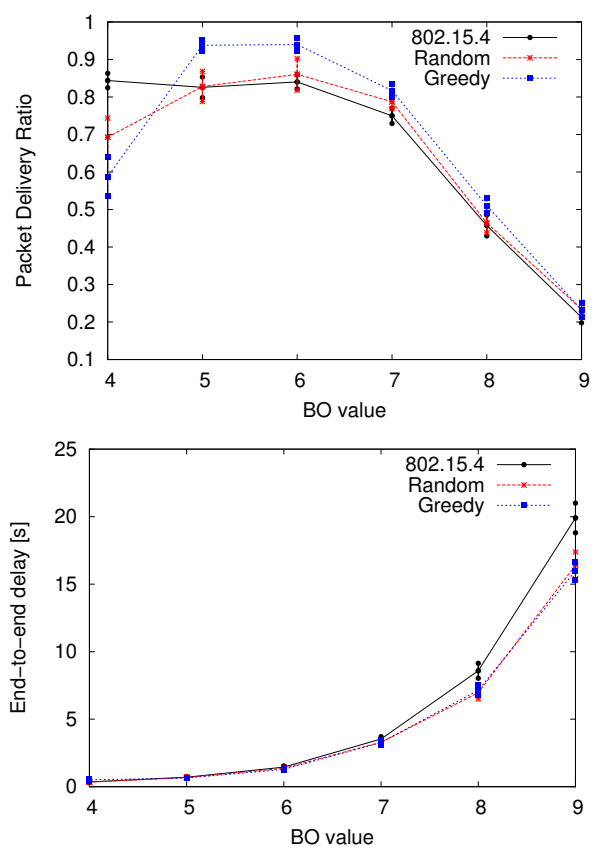

Figure 4. Impact of the duty-cycle $-\mathrm{SO}=2$

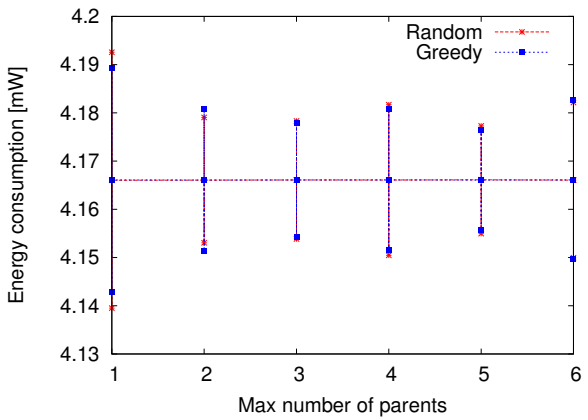

Figure 5. Energy consumption

obviously increases when we increase the number of parents. However, it reaches quickly a maximum of 2 parents on average, the density being low.

Then, we measured the time required before the last node becomes associated (it has a valid parent and it gets a short 16 bits address). Since we maintain the density constant, the diameter increases with the number of nodes: the association time is consequently larger (fig.3(b)). However, it is similar whatever the superframe scheduling algorithm is.

We have finally measured the impact of the number of Beacon-Only-Period slots (fig.3(c)). While the random and greedy strategies are not impacted by the number of slots, IEEE 802.15.4 requires at least 4 slots to reduce the superframe collisions. Too many BOP slots mean a larger overhead, and not enough space for data packets.

We also measured the impact for choosing several parents on the energy consumption (fig. 5). We can verify a node which listens to several parents does not consume significantly more energy: most of the energy consumption is concentrated in maintaining its own superframe. 

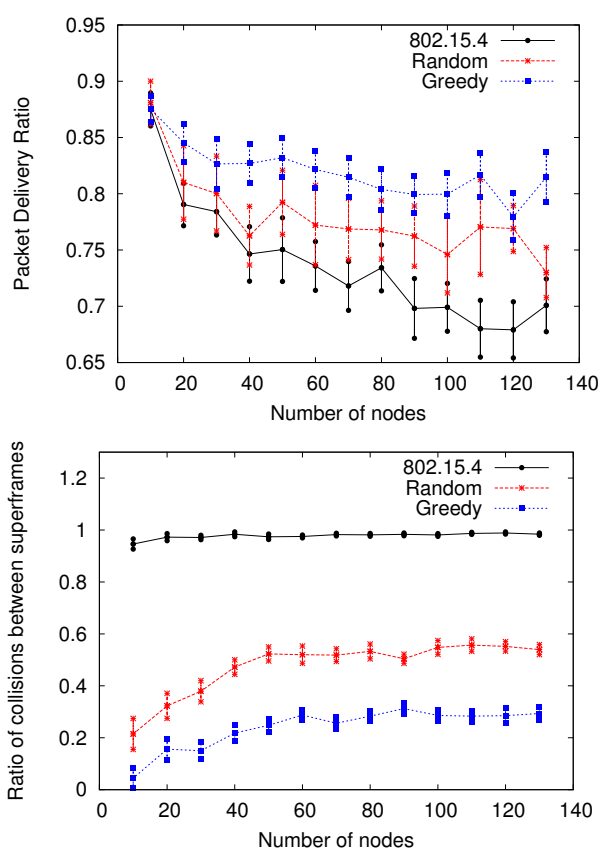

Figure 6. Scalability

\section{Impact of the BO/SO values}

We measured the impact of the number of slots in the scheduling $\left(=2^{B O-S O}\right)$ in fig. 4 . When we have only 4 slots $(\mathrm{BO}=4)$, the diversity is not sufficient to avoid collisions between superframes: the random and greedy algorithms perform worse than the standard. However, as soon as the diversity is sufficient, greedy and random perform better. The packet delivery ratio decreases when $\mathrm{BO}$ increases: the duty cycle decreases, letting less time to transmit and receive packets. We can also verify that the greedy algorithm significantly increases the network capacity, i.e. its ability to forward more packets.

We may also remark the delay increases with BO: a coordinator has on average more time to wait before being in the active part of its superframe. Finally, we can verify that maintaining a DAG in the IEEE 802.15.4 layer helps to reduce the delay: a coordinator has on average less time to wait before entering in the active part of any of its parents.

\section{Scalability}

Finally, we measured the scalability (impact of the network size on the behavior). We measured the PDR in fig.6: it decreases when the number of nodes increases. However, we can remark the greedy superframe selection achieves the best performances: it succeeds to reduce collisions. Inversely, the original IEEE 802.15.4 scheduling performs poorly.

random solution avoids collisions with parents and improves

We also measured the superframe collision ratio: the proportion of coordinators which may suffer from collisions from an interfering coordinator. Reducing this ratio means that we reduce the collisions because of interferences. IEEE 802.15.4 performs very poorly: because the superframe slot depends directly on the depth, almost all the frames collide. This means performances will quickly drop when traffic increases. The significantly the diversity. However, the greedy solution performs better: we reduce the number of collisions by one half compared to a random strategy. Thus, all packet drops will mainly be caused by the IEEE 802.15.4 MAC mechanisms (e.g. too many CCA) and not by interferences: active parts of the superframes are not overlapping for interfering coordinators.

\section{Vi. Conclusion \& Perspectives}

We proposed here to modify the topology of IEEE 802.15.4: adopting a Directed Acyclic Graph structure. A DAG permits to improve the robustness and the delay since a node may choose to have more parents simultaneously. This way it can adopt an opportunistic routing approach: it forwards the packets to the next awake parent. Besides, combining the Beacon-Only Period and the superframe scheduling reduces the collisions while limiting bandwidth wastage when some coordinators have no child in a superframe. Finally, our slot assignment algorithm is very simple, localized, and converges quickly to a stable and accurate assignment. Simulations have proved that modifying the topology and the way IEEE 802.15.4 uses it reduces the beacons and data collisions.

In the future, we plan to explore the impact of realistic experimental collisions on such superframe/BOP slots assignment. We conjecture our algorithm detecting reactively the collisions will be robust to complex interference patterns. We also plan to study how such MAC structure impacts the routing strategy: should the next hop be chased opportunistically or does a multipath scheme improve the reliability and the delay?

\section{REFERENCES}

[1] IEEE 802.15.4-2006 standard, http://www.ieee802.org/15/pub/04.

[2] Francesca Cuomo, Emanuele Cipollone, and Anna Abbagnale. Performance analysis of ieee 802.15.4 wireless sensor networks: An insight into the topology formation process. Computer Networks, 53(18), 2009.

[3] Anna Abbagnale, Emanuele Cipollone, and Francesca Cuomo. A case study for evaluating IEEE 802.15.4 wireless sensor network formation with mobile sinks. In IEEE ICC, Dresden, Germany, June 2009.

[4] Bogdan Pavkovic, Fabrice Theoleyre, and Andrzej Duda. IEEE 802.15.4 and RPL cross-optimization for reliable opportunistic routing in wsn. In ACM MSWiM, Miami, USA, October 2011.

[5] Anis Koubâa, André Cunha, Mário Alves, and Eduardo Tovar. Tdbs: a time division beacon scheduling mechanism for zigbee cluster-tree wireless sensor networks. Real-Time Systems, 40(3):321-354, 2008.

[6] Giuseppe Anastasi, Marco Conti, and Mario Di Francesco. The mac unreliability problem in ieee 802.15 .4 wireless sensor networks. In $A C M$ MSWiM, Tenerife, Spain, 2009. ACM.

[7] IEEE 802.15 WPAN task group 4b (tg4b). http://www.ieee802.org/15/ pub/TG4b.html, September 2004.

[8] B.C. Villaverde, R. De Paz Alberola, S. Rea, and D. Pesch. Experimental evaluation of beacon scheduling mechanisms for multihop ieee 802.15.4 wireless sensor networks. In SENSORCOMM, pages 226-231. IARIA, July 2010.

[9] Panneer Selvan Muthukumaran, Rodolfo de Paz, Rostislav Špinar, and Dirk Pesch. Meshmac: Enabling mesh networking over ieee802.15.4 through distributed beacon scheduling. In AdHocNets, 2009.

[10] I. Rhee, A. Warrier, Jeongki Min, and Lisong Xu. Drand: Distributed randomized tdma scheduling for wireless ad hoc networks. IEEE Transactions on Mobile Computing, 8(10):1384 -1396, oct. 2009.

[11] P. Levis, T. Clausen, J. Hui, O. Gnawali, and J. Ko. The trickle algorithm. IETF rfc 6206, March 2011.

[12] G. Chelius, A. Fraboulet, and E. Fleury. Worldsens: development and prototyping tools for application specific wireless sensors networks. In ACM IPSN, 2007. 